ORIGINAL ARTICLE

\title{
Variations in initial assessment and management of inflammatory bowel disease across Great Britain and Ireland
}

\author{
A Sawczenko, R Lynn, B K Sandhu
}

Arch Dis Child 2003;88:990-994

See end of article for authors' affiliations

......................

Correspondence to: Professor BKSandhu, Department of Gastroenterology, Bristol Children's Hospital, Upper Maudlin Street, Bristol BS2 8BJ, UK;

bhupinder.sandhu@

ubht.swest.nhs.uk

Accepted 7 January 2003
Background: There are no published data from Great Britain and Ireland detailing the initial management of children with inflammatory bowel disease (IBD).

Aims: To prospectively record the initial investigation and treatment of children aged less than 16 years with newly diagnosed IBD.

Methods: For 13 months, between June 1998 and June 1999, 3247 paediatricians, adult gastroenterologists, and surgeons across the UK and Ireland were prospectively surveyed each month and asked to report every newly diagnosed case of childhood IBD. Reporters subsequently completed a postal questionnaire about each case.

Results: A total of 739 new IBD cases were reported from 172 institutions. Significant variations were observed in the investigation and treatment of these cases, when examined by number of cases reported per institution, or by the specialists providing care. There were wide regional variations in the proportion of children having access to paediatric gastroenterology services. Overall, one third of children received care from an adult service, and a tenth care exclusively from an adult gastroenterologist. Children with Crohn's disease who had some or all of their care from adult services were more likely to receive systemic steroids and less likely to receive dietary therapy; those with ulcerative colitis were more likely to receive rectal steroids and to have surgery. Height and weight were also less likely to be recorded in those whose care involved adult services.

Conclusion: Current specialist provision, and initial investigation and treatment of IBD, is heterogeneous. Optimisation of care is likely to be achieved by greater access to specialist paediatric gastroenterology services for all those with suspected IBD. listis nflammatory bowel disease (IBD) in those aged less than 16 years, presents at a median age of $12-13$ years. ${ }^{12}$ Studies suggest that the incidence has probably increased..$^{1-4}$ Ideally children, no matter where they live, should receive the same high standard of care, including access to appropriate specialists. Anecdotally however there appears to be considerable variation in the nature of the specialist care provided across Great Britain and Ireland, with much care being provided by adult services. This may be of relevance, because as well as important psychological and family issues, childhood onset IBD is characterised by physiological and pathological differences from adult onset disease. In particular, the disease may be more extensive, with the potential for significant growth impairment resulting in short adult stature..$^{5-9}$

Modern guidelines on the provision of tertiary services for children and adolescents emphasise the importance of care being provided in an age appropriate setting. ${ }^{10}$ In order to learn more about the current provision of services for children with IBD, we undertook a prospective survey of newly diagnosed cases across Great Britain and Ireland. ${ }^{1}$ This paper describes significant variation in the specialists providing care, variation in initial investigations undertaken, and variation in the treatment given to new cases of childhood IBD.

\section{METHODS}

For 13 months between 1 June 1998 and 30 June 1999 new cases of childhood inflammatory bowel disease (IBD) were prospectively identified in Great Britain and Ireland as previously described. ${ }^{1}$ This survey aimed to be as comprehensive as possible by collecting data on all known incident cases of IBD. A total of 1852 paediatric specialists were surveyed through the well validated British Paediatric Surveillance Unit (BPSU) of the Royal College of Paediatrics and Child Health, ${ }^{11}$ and through the Paediatric Register of Inflammatory Bowel Disease (PRIBD), set up in 1997 to record data from 53 UK centres. A total of 1395 adult gastroenterologists and surgeons were prospectively surveyed through the newly established British Society of Gastroenterology Research Unit (BSGRU).

Participants were mailed a surveillance card each month to report new cases of IBD diagnosed in individuals aged less than 20 years in the preceding month. If a case was reported, a detailed or "full" postal questionnaire was then sent out by the BSPU to gather further information about each case. The BSGRU sent out an initial "abbreviated" questionnaire to establish the diagnosis and then followed this up with a longer secondary questionnaire, which contained fewer fields than the "full" BPSU questionnaire. Some paediatricians preferred to report details only to the Paediatric Register of Inflammatory Bowel Disease (PRIBD). Cases were reported to the PRIBD on a monthly basis using an abbreviated

Abbreviations: 5-ASA, 5-aminosalicylate; BPSU, British Paediatric Surveillance Unit; BSGRU, British Society of Gastroenterology Research Unit; $C D$, Crohn's disease; $\mathrm{Cl}$, confidence interval; IBD, inflammatory bowel disease; IC, indeterminate colitis; OGD,

oesophagoduodenoscopy; PRIBD, Paediatric Register of Inflammatory Bowel Disease; RR, relative risk; UC, ulcerative colitis 
questionnaire with similar, though fewer, fields to the full BPSU questionnaire. Although most centres reported to both studies we were aware that a few reporters did not, and we therefore accessed the PRIBD database at the end of the BPSU and BSGRU surveys. Details of height and weight were not collected for those cases identified via the PRIBD.

England is the most populous country in the United Kingdom. The BPSU divides it into 14 regions corresponding to the regional health authorities prior to the 1994 reorganisation of the National Health Service. In addition we undertook analyses by country-that is, between England, Scotland, Wales, Northern Ireland, and the Republic of Ireland.

\section{RESULTS}

The mean monthly return of reporting cards was $94 \%$ in the BPSU survey and $46 \%$ in the BSGRU survey. ${ }^{1}$ The BPSU, BSGRU, and PRIBD received 972, 641, and 422 reports respectively during the study period. Thirty seven reports were excluded because they were mistaken notifications, 538 were duplicates, 584 confirmed IBD cases were aged over 16.0 years at diagnosis, 61 cases were diagnosed outside the study period, two cases were foreign, 28 reports had a final diagnosis not of IBD, and in 46 instances, despite repeated attempts, follow up of the initial card report was not possible.

There were thus 739 incident IBD cases aged less than 16 years diagnosed between 1 June 1998 and 30 June 1999 in Great Britain and Ireland: 431 Crohn's disease, 11 orofacial granulomatosis, 86 indeterminate colitis, and 211 ulcerative colitis cases. The BPSU, BSGRU, and PRIBD surveys contributed $88 \%, 8 \%$, and $4 \%$ of these cases respectively.

A complete BPSU questionnaire was returned in 83\% (615/ 739) of cases and an "abbreviated" questionnaire in $17 \%$ (124/739) of cases, either a partially completed BPSU questionnaire, a BSGRU questionnaire, or a PRIBD questionnaire. From these responses details of the specialists providing care were available in $94 \%(696 / 739)$ of cases and details of treatment in $84 \%(623 / 739)$ of cases. Details of what investigations had been undertaken were available for most children, for example 86\% (642/739) of cases had colonoscopy. There were varying and slightly smaller figures for other investigative modalities.

\section{"Size" of reporting centre}

Reports were received from 172 centres. Centres were empirically divided into "small" centres ( $1-5$ reports), "medium" centres (6-10 reports), and "large" centres (>11 reports) depending on the numbers of incident cases reported during the study. A total of $34 \%(250 / 739)$ of cases were reported from "small" centres, 15\% (111/739) from "medium" sized centres, and 51\% (378/739) from "large" centres.

Thus the majority of centres reported either one or two cases $(64 \%, 110 / 172)$. The largest centre reported 43 new cases over 13 months. Twenty seven teaching hospitals reported 53\% (391/739) of cases and 145 district general hospitals $47 \%$ (348/739) of cases.

There were significant regional variations across England in the proportion of cases reported from small, medium, and large centres (ranges $7-87 \%, 7-39 \%$, and 3-93\% respectively; all $p<0.001, \chi^{2}$ test). While some regions appeared to have an obvious "hub" to which nearly all cases were referred, most did not.

\section{Investigations}

A greater proportion of all IBD cases reported from large centres had an oesophagoduodenoscopy (OGD), colonoscopy, or barium follow through and a smaller proportion a barium enema or sigmoidoscopy, compared to those cases reported from small centres (RR 2.74 (95\% CI 2.12 to 3.54), RR 1.37
(95\% CI 1.24 to 1.51 ), RR 1.28 (95\% CI 1.11 to 1.47 ), RR 0.51 (95\% CI 0.28 to 0.92 ), and RR 0.45 (95\% CI 0.34 to 0.58 ) respectively, all $\mathrm{p}<0.001)$. Cases reported from large centres were less likely to have had "dual investigation" by both colonoscopy and sigmoidoscopy compared to those from small centres (RR 0.68 (95\% CI 0.47 to 0.97), p $=0.035$ ).

These associations remained significant when small, medium, and large centres were compared.

\section{Treatment}

Cases of Crohn's disease were more likely to be treated with diet and less likely to be treated with systemic steroids in large and medium centres, compared to small centres (fig 1), and were also less likely to receive rectal 5 -aminosalicylate (5-ASA) preparations $\left(\mathrm{p}<0.01, \chi^{2}\right.$ test $)$. Comparison between large, medium, and small centres showed that the association between size of centre and steroid use remained but that the association for diet did not reach significance (fig 1). In this analysis the association with rectal 5-ASA became non-significant, but that for rectal steroids became significant ( $\mathrm{p}=0.014, \chi^{2}$ test), with the greatest use being reported from small centres.

For cases of ulcerative colitis, significant variations were observed in the use of systemic 5-ASA by centre size, comparing large centres with small centres or large and medium centres with small centres (RR 0.78 (95\% CI 0.66 to $0.92), p=0.006$; and RR 0.83 (95\% CI 0.72 to 0.96 ), $\mathrm{p}=0.027$, respectively).

Rectal steroids were used less for all types of IBD in large centres compared to small centres, and this was particularly so for Crohn's disease (RR 0.44 (95\% CI 0.22 to 0.89 ), $\mathrm{p}=0.02$; and RR 0.14 (95\% CI 0.03 to 0.67 ), $\mathrm{p}<0.01$, respectively).
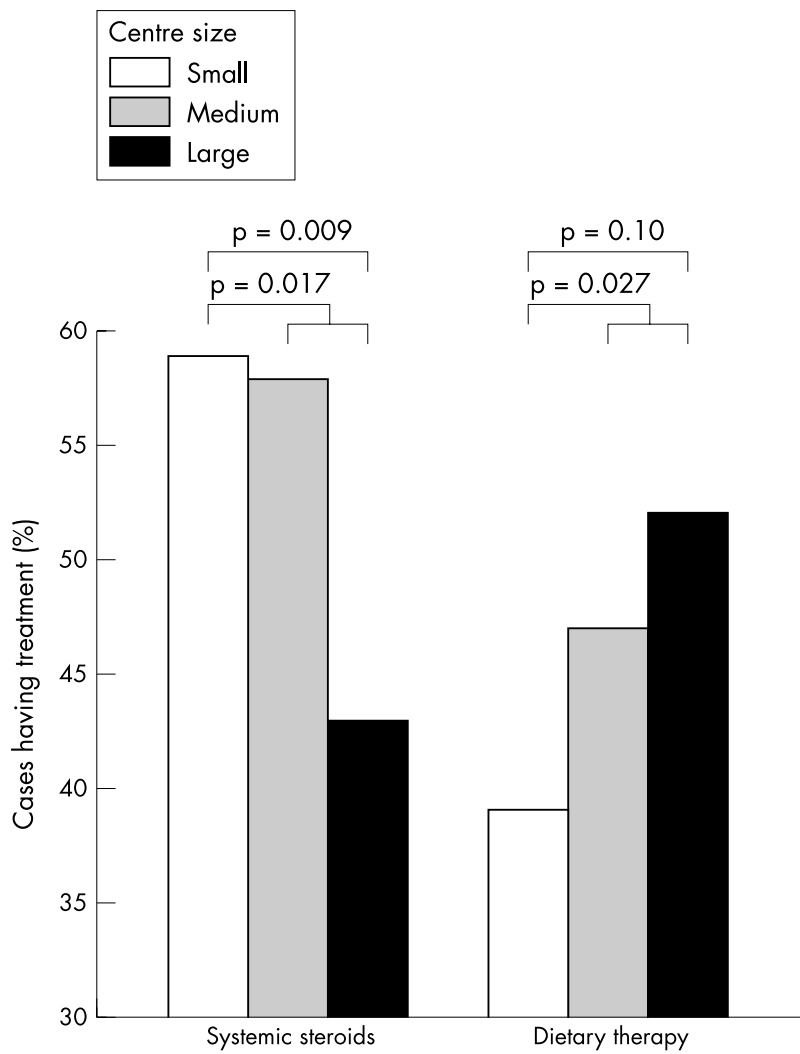

Figure 1 Treatment of Crohn's disease by reporting centre size. 
Table 1 Specialist teams by centre size

\begin{tabular}{|c|c|c|c|c|c|c|c|c|}
\hline \multirow[b]{2}{*}{ Centre size } & \multicolumn{4}{|c|}{ One specialist } & \multicolumn{3}{|c|}{ Two specialists } & \multirow[b]{2}{*}{$\begin{array}{l}3 \text { or more } \\
\text { specialists }\end{array}$} \\
\hline & Paed GI & Paed & Adult GI & Sur $(\mathbf{P})$ & $\begin{array}{l}\text { Paed GI + } \\
\text { another }\end{array}$ & $\begin{array}{l}\text { Adult GI + } \\
\text { another }\end{array}$ & $\begin{array}{l}\text { Other } \\
\text { combinations }\end{array}$ & \\
\hline Small & 10 & 19 & 58 & 1 & $53^{*}$ & 58 & 18 & 31 \\
\hline Medium & 26 & 9 & 5 & & 37 & 7 & 10 & 12 \\
\hline Large & 165 & 1 & 2 & & $142^{\text {** }}$ & 9 & 2 & 20 \\
\hline
\end{tabular}

$\mathrm{Gl}$, gastroenterologist; Sur (P), paediatric surgeon.

Cases where "adult Gl" was the second doctor: *includes 7 cases, **includes 4 cases.

\section{Type of specialist}

The majority of children received care from more than one type of specialist (table 1). There were significant differences in the composition of the teams providing care, depending on the "size" of the reporting institution (fig 2).

\section{Involvement of adult services}

Thirty per cent (211/696) of children received care from either an adult gastroenterologist or an adult surgeon, and a further $3 \%$ (18/696) from both an adult surgeon and an adult gastroenterologist. Thus 33\% (229/696) of children had been involved with adult services; their median age was 13.6 years compared to 12.1 years for those whose care had not involved an adult gastroenterologist and/or surgeon $(p<0.001$, Mann Whitney).

Of those children cared for by adult services, 65 were reported to have been managed solely by an adult gastroenterologist; the median age of this group was greater than that of children whose care was shared with paediatric services (median age 15.0 years versus 13.0 years, $p<0.001$, Mann-Whitney); however, it included two children aged less than 5 years and three aged between 5 and 10 years.

\section{Investigations}

If care had involved an adult gastroenterologist and/or an adult surgeon, children were more likely to have had a sigmoidoscopy or barium enema and less likely to have had a colonoscopy, OGD, or barium follow through (RR 2.07 (95\% CI 1.64 to 2.61 ), RR 2.94 (95\% CI 1.71 to 5.07 ), RR 0.70 (95\% CI 0.62 to 0.78 ), RR 0.35 (95\% CI 0.26 to 0.46$)$, RR 0.79 ( $95 \%$ CI 0.68 to 0.91$)$ respectively, all $\mathrm{p}<0.001$; table 2 ). Conversely if a paediatric gastroenterologist had been involved in initial management, children were more likely

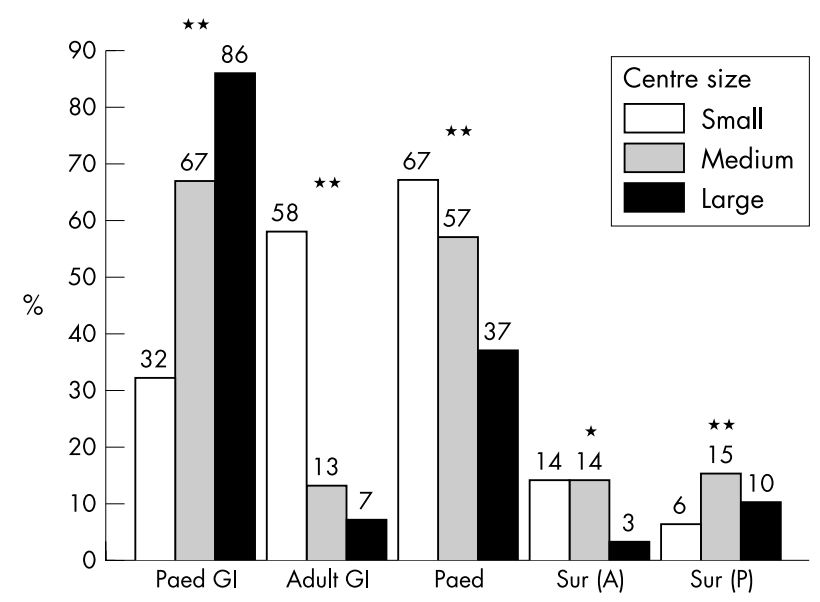

Figure 2 Specialist involvement by centre size. Gl, gastroenterologist; Sur $(A)$, adult surgeon; Sur $(P)$, paediatric surgeon. ${ }^{*} p<0.05$, ${ }^{* *} p<0.001, \chi^{2}$ test. to have had a colonoscopy, OGD, and barium follow through and less likely to have had a sigmoidoscopy or barium enema (RR 1.47 (95\% CI 1.30 to 1.66 ), RR 3.64 (95\% CI 2.59 to 5.11 ), RR 1.29 (95\% CI 1.11 to 1.49$)$, RR 0.49 (95\% CI 0.39 to 0.61 ), RR 0.34 (95\% CI 0.20 to 0.58 ) respectively, all p $<0.001)$. The combination of sigmoidoscopy and colonoscopy was more likely to have taken place if care had involved an adult surgeon (RR 2.18 (95\% CI 1.30 to 3.63 ), p $<0.01$ ).

\section{Medical treatment}

Children with Crohn's disease whose care involved an adult gastroenterologist were more likely to have received systemic steroids and less likely to have received dietary therapy at diagnosis (RR 2.19 (95\% CI 1.34 to 3.57), p = 0.001; and RR 0.73 (95\% CI 0.51 to 1.03 ) $\mathrm{p}=0.047$, respectively); those with ulcerative colitis were more likely to have received rectal steroids, and to have been treated with a low fibre or restriction diet (RR 1.89 (95\% CI 1.14 to 3.16$), \mathrm{p}=0.02$; and RR 4.36 (95\% CI 1.29 to 14.75 ), p $=0.01$, respectively). In contrast, if initial care involved a paediatric gastroenterologist, children with Crohn's disease were less likely to receive systemic steroids (RR $0.84 \quad(95 \%$ CI 0.75 to 0.94$)$, $\mathrm{p}=0.0022)$, and those with ulcerative colitis were less likely to receive rectal steroids (RR 0.52 (95\% CI 0.29 to 0.94 ), $\mathrm{p}=0.031$ ).

\section{Surgery}

All four cases of ulcerative colitis that had had surgery were treated by adult services ( $p<0.01$, Fisher's exact test).

\section{Histology}

Specimens for histology were reported as not being taken in 29/739 (4\%) cases; 24 cases of Crohn's disease, four of ulcerative colitis, and one indeterminate colitis. This was more likely to have occurred if an adult gastroenterologist and/or surgeon had been involved in care (RR 7.16 (95\% CI 3.10 to 16.51$), \mathrm{p}<0.001)$.

\section{Height and weight}

Height and weight were less likely to have been recorded if care had involved an adult gastroenterologist (RR 0.86 (95\% CI 0.78 to 0.96 ) p $<0.01$; and RR 0.93 (95\% CI 0.86 to 1.01 ), $\mathrm{p}=0.047$ ). This was particularly the case if care had been provided solely by an adult gastroenterologist (RR 0.38 (95\% CI 0.26 to 0.55 ); and RR 0.57 (95\% CI 0.45 to 0.73 ), respectively; both $\mathrm{p}<0.001$ ).

\section{Country and region}

A total of 575 cases were reported from England, 71 from Scotland, 34 from Wales, 16 from Northern Ireland, and 43 from the Republic of Ireland (ROI). There were significant variations between countries in the proportion of children receiving care from a paediatric gastroenterologist, or an adult gastroenterologist and/or surgeon (fig 3), with similar differences between the 14 English regions (ranges 14-92\%, $\mathrm{p}<0.001$; and $8-71 \%, \mathrm{p}<0.001$, respectively, $\chi^{2}$ test $)$. 
Table 2 Initial investigation

\begin{tabular}{lccccc}
\hline & All IBD (\%) & CD (\%) & IC (\%) & UC (\%) & \\
\hline OGD & 48 & 67 & 42 & 31 & $* *$ \\
BaFT & 65 & 78 & 59 & 38 & NS \\
Colonoscopy & 82 & 82 & 84 & 78 & NS \\
Barium enema & 8 & 7 & 6 & 11 & $* *$ \\
Sigmoidoscopy & 30 & 23 & 39 & 41 & $* *$ \\
WCC & 28 & 37 & 25 & 17 & NS \\
Colonoscopy + & 16 & 14 & 23 & 20 & $* *$ \\
sigmoidoscopy & 16 & 21 & 14 & 5 & \\
BaFTT + WCC & 16 & & & & \\
\hline
\end{tabular}

$C D$, Crohn's disease; IC, indeterminate colitis; UC, ulcerative colitis; OGD, oesophagoduodenoscopy; BaFTT + WCC, barium follow through and radiolabelled white cell scan. ${ }^{* *} \mathrm{p}<0.001, \chi^{2}$ test.

Comparison between the five countries showed significant variations in the proportion of children receiving specific investigations or treatments, with even greater variations between the English regions (data not shown).

\section{Current treatment practice}

The majority of children with Crohn's disease received an oral 5-ASA preparation with no clear "first choice" of systemic steroids or diet for inducing remission (table 3). (A small number of children received both of these therapies.) Polymeric feeds were more commonly used than elemental or "semi" elemental feeds. There was no relation between site of disease activity and use of systemic steroids; however, diet was more likely to have been used if there was disease activity in the terminal ileum, and an oral 5-ASA preparation if there was disease activity in the left colon (RR 1.21 (95\% CI 1.05 to 1.40 ), $\mathrm{p}=0.008$; and RR 1.23 (95\% CI 1.02 to 1.48 ), $\mathrm{p}=0.022$, respectively).

Systemic steroids and oral 5-ASA preparations were the mainstay of treatment for indeterminate colitis and ulcerative colitis, although nearly a fifth of children did not receive oral 5-ASA preparations. Polymeric or elemental diet was used in nearly a fifth of children with indeterminate colitis.

\section{DISCUSSION}

This prospective population based survey is the largest such study of childhood inflammatory bowel disease to date and the first to document provision of care across Great Britain and Ireland. ${ }^{1}$ The study found considerable variation in care

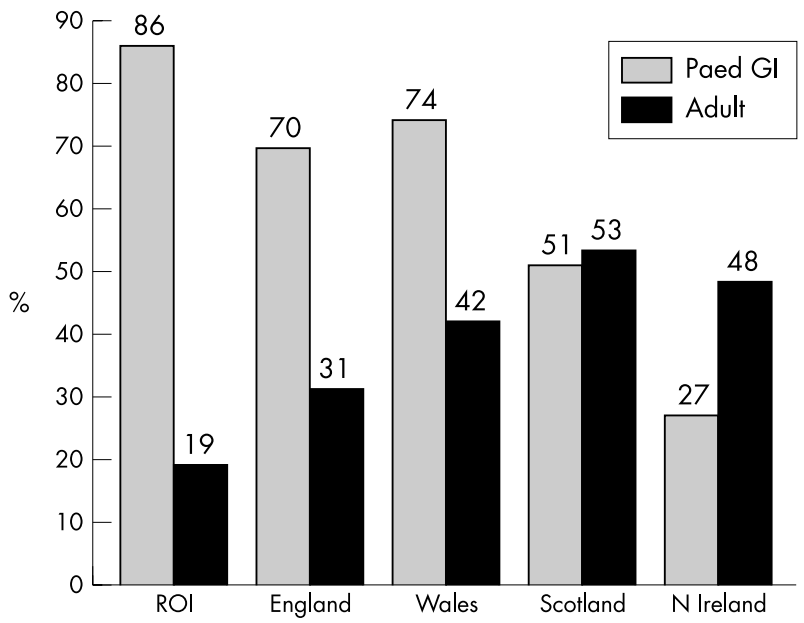

Figure 3 Selected specialist involvement by country. Paed GI paediatric gastroenterologist; adult, adult gastroenterologist and/or adult surgeon; ROI, Republic of Ireland. Variations between countries for Paed $G$ and Adult, both $p<0.001, \chi^{2}$ test.
Table 3 Initial treatment

\begin{tabular}{lcccc}
\hline & All IBD (\%) & CD (\%) & IC (\%) & UC (\%) \\
\hline Systemic steroids & 56 & 50 & 56 & 68 \\
Oral 5-ASA & 69 & 62 & 81 & 79 \\
Rectal steroids & 9 & 4 & 13 & 20 \\
Rectal 5-ASA & 3 & 2 & 1 & 6 \\
Azathioprine & 6 & 5 & 7 & 8 \\
Cyclosporin & 1 & 1 & 1 & 2 \\
Metronidazole & 12 & 15 & 14 & 6 \\
Surgery & 3 & 5 & & 2 \\
Diet & 33 & 47 & 22 & 9 \\
\hline CD, Crohn's disease; IC, indeterminate colitis; UC, ulcerative colitis.
\end{tabular}

in terms of initial investigations and treatment when examined either by the number of cases reported by an institution or by the type of specialist gastrointestinal input (adult or paediatric gastroenterologist).

At present two thirds of children aged less than 16 years have some input from a paediatric gastroenterologist at diagnosis, but one tenth have their initial management provided solely by an adult gastroenterologist, and a third have some of their care provided by adult services. It is likely that there are differences in the provision of specialist paediatric gastroenterology services in Great Britain and Ireland. This survey was not designed to determine whether the observed regional variation in use of paediatric gastroenterology services was due to lack of provision of such services or failure to refer to them.

Most children (82\%) had a colonoscopy at the time of diagnosis; however, nearly one third $(30 \%)$ had a sigmoidoscopy and $16 \%$ both examinations. Sigmoidoscopy was more likely to be undertaken and colonoscopy less likely to be undertaken if care had involved an adult service. It has been shown in children that an incomplete colonoscopy reduces the chances of establishing an histological diagnosis. ${ }^{16}$ One possible explanation is resourcing difficulties, ${ }^{17}$ although differences in acceptable management practices between adult and paediatric gastroenterologists is also likely.

The greatest variation in treatment was noted for cases of Crohn's disease, with clear differences between adult and paediatric practice. Cases whose management involved an adult gastroenterologist were more likely to receive systemic steroids and less likely to receive dietary therapy. Such differences probably reflect the concern among paediatricians regarding the growth inhibiting effects of steroids, as well as the proven efficacy of enteral feeding in paediatric studies in contrast to the disappointing efficacy in adult studies. ${ }^{12}{ }^{13}$ The sole use of enemas in children, as noted among those cared for by adult gastroenterologists, may reflect adult practice and be less appropriate because colitis is rarely confined solely to the rectum or left colon in children. Overall this 
study also confirms the continuing wide usage of systemic steroids for both childhood Crohn's disease and ulcerative colitis. ${ }^{14}$ While a small proportion of children will inevitably require surgery, the finding of a higher rate of surgery among adult services at the onset of disease remains unexplained.

Failure to record growth parameters, particularly for those not presenting to a paediatrician, has been previously identified; this study confirms that this continues to occur. ${ }^{15}$

A recent report commented that a significant minority of childhood IBD cases were inappropriately or inadequately investigated and treated. ${ }^{18}$ It is noteworthy that in the current study the majority of units reported only one or two new cases of IBD over a 13 month period. Of the "small" institutions (reporting 1-5 cases), some appeared to have a clear lead clinician reporting all new cases, but in most institutions each report came from a different clinician (data not shown). This suggests that in many, if not the majority, of institutions there is no designated care pathway for the management of childhood IBD.

With the rapid changes in the training and manning of paediatric departments it remains to be determined whether a "hub and spoke" or "managed clinical network" can ensure that children receive high quality care in an age appropriate setting. In a number of paediatric medical and surgical subspecialties, as well as those that cross boundaries, centralisation to specialist care has been recognised to provide optimal care. ${ }^{19-25}$ Currently there is no consensus that such centralisation should be applied to gastroenterology services for children.

The key issue arising from this study appears to be how to ensure high quality care for those living some distance from specialist centres with a model that takes into account the number of specialists and journey times of families. The British Society of Paediatric Gastroenterology, Hepatology and Nutrition recommends in its guide for purchasers of children's services that all those with suspected IBD should be managed by a specialist paediatric gastroenterology unit. $^{26}$

\section{ACKNOWLEDGEMENTS}

This work would not have been possible without the support of the members of the Royal College of Paediatrics and Child Health, the members of the British Society of Gastroenterology and others who reported cases. We thank them. It was supported by the British Society of Paediatric Gastroenterology, Hepatology and Nutrition and we would like to thank the society and its members.

We wish to especially thank Dr Angus Nicoll of the British Paediatric Surveillance Unit of the Royal College of Paediatrics and Child Health, Ms Sarah Mian and Professor Richard Logan of the British Society of Gastroenterology Research Unit, Dr Tim Card of the Department of Public Health Epidemiology, University of Nottingham, Dr Huw Jenkins and Dr David Casson of the Paediatric Register of Inflammatory Bowel Disease, and Prof Chris Taylor of the Sheffield Children's Hospital.

The study was funded by donations from the National Association of Crohn's and Colitis, SHS International, and the Bristol Paediatric Gastroenterology Fund.

\section{Authors' affiliations}

A Sawczenko, B K Sandhu, Department of Gastroenterology, Bristol

Children's Hospital, Bristol, UK

R Lynn, British Paediatric Surveillance Unit, Royal College of Paediatrics and Child Health, London, UK

\section{REFERENCES}

1 Sawczenko A, Sandhu BK, Logan RFA, et al. Prospective survey of childhood inflammatory bowel disease in the British Isles. Lancet 2001;357:1093-4.

2 Barton JR, Gillon S, Ferguson A. Incidence of inflammatory bowel disease in Scottish children between 1968 and 1983, marginal fall in ulcerative colitis, three-fold rise in Crohn's disease. Gut 1989;30:618-22.

3 Cosgrove M, Al-Atia RF, Jenkins HR. The epidemiology of paediatric inflammatory bowel disease. Arch Dis Child 1996:74:460-1.

4 Armitage E, Drummond H, Ghosh S. Incidence of juvenile-onset Crohn's disease in Scotland. Lancet 1999;353:1496-7.

5 Engstrom I, Lindquist BL. Inflammatory bowel disease in children and adolescents: a somatic and psychiatric investigation. Acta Paediatr Scand 1991:80:640-7.

6 Kirschner BS. Differences in the management of inflammatory bowel disease in children and adolescents compared to adults. Netherlands J Med 1998;53(6):S13-18

7 Puntis J, McNeish AS, Allan RN. Long term prognosis of Crohn's disease with onset in childhood and adolescence. Gut 1984;25:329-36.

8 Griffiths AM, Nguyen P, Smith C, et al. Growth and clinical course of children with Crohn's disease. Gut 1993;34:939-43.

9 Hildebrand H, Karlberg J, Kristiansson B. Longitudinal growth in children and adolescents with inflammatory bowel disease. J Pediatr Gastroenterol Hepatol Nutr 1994;18:165-73.

10 BPA. Tertiary services for children and young people. British Paediatric Association, 1995.

11 Hall SM, Nicoll A. The British Paediatric Surveillance Unit-a pioneering method for investigating the less common disorders of childhood. Report of a seminar held in June 1995. Child Care Health Develop 1998;24:129-43.

12 Sanderson IR, Udeen S, Davies PS, et al. Remission induced by an elemental diet in small bowel Crohn's disease. Arch Dis Child 1987;62:123-7.

13 Heuschkel RB, Menache CC, Megerin JT et al Enteral nutrition and corticosteroids in the treatment of acute Crohn's disease in children. J Pediatr Gastroenterol Nutr 2000;31:8-15.

14 Barton JR, Ferguson A. Clinical features, morbidity and mortality of Scottish children with inflammatory bowel disease. QJM 1990;75:423-39.

15 Ghosh S, Drummond HE, Ferguson A. Neglect of growth and development in the clinical monitoring of children and teenagers with inflammatory bowel disease: review of case records. BMJ 1998;317:120-1.

16 Holmquist L, Rudic N, Ahren C, et al. The diagnostic value of colonoscopy compared with rectosigmoidoscopy in children and adolescents with symptoms of chronic inflammatory bowel disease of the colon. Scand J Gastroenterol 1988;23:577-84.

17 Macfarlane B, Leicester R, Romaya C, et al. Colonoscopy services in the United Kingdom. Endoscopy 1999;31:409-11.

18 Spray C, Debelle GD, Murphy MS. Current diagnosis, management and morbidity in paediatric inflammatory bowel disease. Acta Paediatr $2001 ; 90: 400-5$.

19 Arul GS, Spicer RD. Where should paediatric surgery be performed? Arch Dis Child 1998:79:65-70.

20 Baumer JH, Hunt LP, Shield JP. Audit of diabetes care by caseload. Arch Dis Child 1997;77:102-7.

21 Mahadeva R, Webb K, Westerbeek RC, et al. Clinical outcome in relation to care in centres specialising in cystic fibrosis: cross sectional study. BMJ 1998;316:1771-5

22 Sharples A, O'Neill M, Dearlove O. Impact of specialised paediatric retrieval teams. Children are still transferred by non-specialist teams. BMJ 1996;312:120-1

23 Pearson G, Shann F, Barry P, et al. Should paediatric intensive care be centralised? Trent versus Victoria. Lancet 1997;349:1213-17.

24 McKiernan PJ, Baker AJ, Kelly DA. The frequency and outcome of biliary atresia in the UK and Ireland. Lancet 2000;355:25-9.

25 Field D, Hodges S, Mason E, et al. Survival and place of treatment after premature delivery. Arch Dis Child 1991:66:408-10.

26 BSPGN. A guide for purchasers. Paediatric gastroenterology and nutrition services The British Society of Paediatric Gastroenterology and Nutrition, 1996. 